\title{
GRID Technologies => ‘Education’ = ‘Distance Education'
}

\author{
Michalis Xenos ${ }^{1,2}$, Bill Vassiliadis ${ }^{1}$, Athanassios Skodras ${ }^{1}$ \\ ${ }^{1}$ Hellenic Open University, School of Sciences \& Technology, Patras, Greece \\ ${ }^{2}$ R.A. Computer Technology Institute, Patras, Greece \\ $\{x e n o s, b b$, skodras\}@eap.gr
}

\begin{abstract}
This paper discusses the new possibilities that Grid technologies create in education, presents current learning paradigms and makes a prediction about the way in which Grid technologies may affect the future of education. The case of the Hellenic Open University (HOU) is presented and the current educational technologies and tools used are illustrated. The paper also presents a scenario for the utilization of Grid technologies at HOU and discusses the challenges that such infrastructure creates, as well as the services that it can enable for HOU students.
\end{abstract}

Keywords: Grid, Education, Collaboration, Virtual Scientific Experiments

\section{INTRODUCTION}

The Grid is emerging as a major enabler for networked organizations [1]. Although several of the enabling technologies are at a cusp in their development, the Grid has matured to its third generation of development adopting a service-oriented approach. A more holistic view of business infrastructures is supported and services are metadata enabled and ontologically principled. Some autonomic features are exhibited as well.

Traditionally, Grid computing has addressed the needs of long-running scientific computations submitted as batch jobs. Long-running batch jobs can be distributed across several nodes in a Grid and executed in parallel, resulting in shorter execution times. The benefits of Grid computing extend to the provision of resource virtualization already supported by developing standards of the Open Grid Services Architecture (OGSA) [2]. Their use can shift the boundary of traditional networked models out to dynamically include and exclude elements of other organizations, thus increasing management and economical complexity. The use of Grid services permits the direct or indirect participation of organizations in large cooperation networks, which constitute very large dynamic systems. In this context, services are viewed as Grid- services that allow, amongst others, dynamic service instantiation and interaction, lifetime management and handling of complex activity requests from users [3].

Nevertheless, in the case of networked business models, and thus from a virtual organization (VO) point of view, traditional technologies such as virtual private networks and Intranets/Extranets based on HTTP provide just an infrastructure for their basic functionality. VOs need to be more dynamic, agile and able to extend automatically. The Grid reflects this philosophy by enabling on demand services, a step towards Utility/On demand Computing. A number of projects have proposed the use of Grid resources as computation utility include Globus [9], NetSolve [10] and Cactus [11].

The vision of integrating Grid and Web service concepts and technologies has been proposed as the OGSA. With efforts in OGSA implementation and standardization, application services are expected to be widely deployed and more interoperable [3].

Conceptually, the Grid can be thought of in terms of three layers: the computational/data Grid, the information Grid and the knowledge Grid (Figure 1).

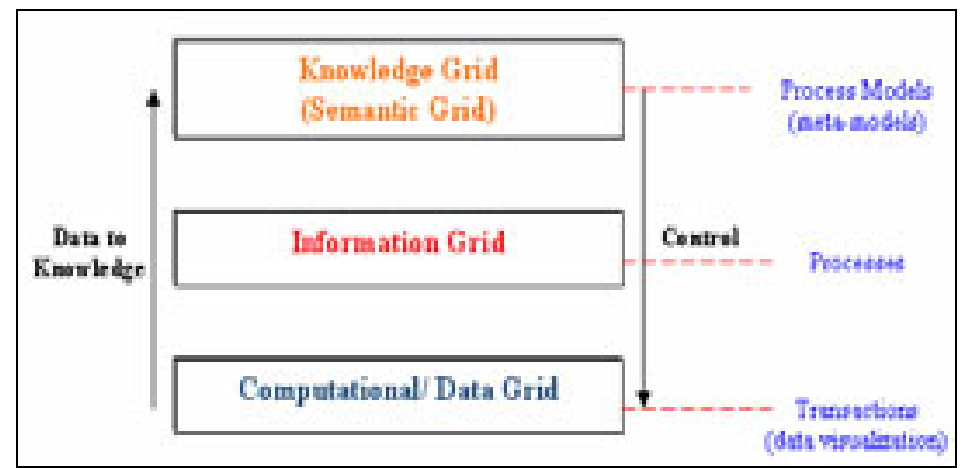

FIGURE 1: Three layer Grid abstraction 
The bottom layer is the computational and data grid: the computer hardware and data networks upon which the work will be conducted. Above this lies the 'information grid': the databases of information to be accessed by the hardware, and systems for data manipulation. On top lies the 'knowledge grid', where high-level applications mine the data for the knowledge that can form the basis of semantic understanding and intelligent decision-making.

A data/computational grid forms the fabric of the Grid to provide raw computing power, high-speed bandwidth and associated data storage in a secure and auditable way. Diverse resources are represented as a single computer so the way that computational resources are allocated, scheduled and executed, and the way that data is shipped between processing resources, is handled here.

A knowledge grid uses knowledge based methodologies and technologies for responding to high-level questions and finding the appropriate processes to deliver answers in the required form. This last layer includes data mining, machine learning, simulations, ontologies, intelligent portals, workflow reasoning and Problem Solving Environments for supporting the way knowledge is acquired, used, retrieved, published and maintained. A knowledge grid should provide intelligent guidance for decision makers and hypothesis generation.

Despite the provoking implication of its title, this paper does not argue that Grid technologies will eventually turn every form of education into distant. It does argue, however, that the use of Grid technologies can turn a number of educational tasks normally conducted at a specific place (i.e. classroom) so far into distant ones (i.e. anywhere) and that this may gradually alter the way we think about education.

The rest of the paper is structured as follows: Following this brief literature review, section 2 discusses the new potential that the utilization of Grid technologies creates in education and makes a prediction about the way in which Grid technologies may affect the future of education. Section 3 briefly presents the HOU case and the distance education technologies and tools currently used and proposes a scenario for the utilization of Grid technologies by the HOU. Finally, section 4 outlines the major conclusions of the paper.

\section{THE GRID-DRIVEN FUTURE OF EDUCATION}

In this section and throughout the rest of the paper the term 'Learner' will be used for anyone that learns something, formally or informally, either under a well-organized framework (i.e. school, university), or occasionally (i.e. in his house, while waiting for the bus, etc.). The term 'Tutor' is used to define the notion of tutoring in general and not a specific person (i.e. teacher, professor); in this context a tutor may also be a student offering an explanation to a fellow student.

\subsection{The evolution of modern education}

The utilization of currently available communication and information technologies has turned traditional location based education into location independed. No one will deny that location (i.e. school, university) has a strong social impact on the learner, but the learner may still learn and study in a number of 'non-educational' locations. Of course, location based education is still required in certain cases such as laboratories, complex experiments conduction, etc.

Today learning is equivalent to searching for sources and selecting the appropriate source to study from, while the role of teachers and professors is becoming more and more that of a consultant (tutor) rather than that of the traditional knowledge communicator. This is mostly because the plethora of sources available on the Internet makes the selection of the appropriate source a rather difficult task, which is why the role of the tutor is important. Nevertheless, despite the wide availability of online sources, important elements of the learning procedure such as practicing and collaborating have not been enabled yet by current technologies and existing infrastructures. The same applies in the case of complex experiments conduction and data processing, as learners today need to actively participate in numerous experiments that allow them to practice on what they study. Such experiments may not be available everywhere, or may require computing power that is not available to every learner. Learners also need to access large volumes of data, most times distributed in many locations.

Most of all, today's learners need a variety of services available on demand that can be used/accessed from their environment. It must also be noted that in applied sciences, experimentation has a central role in teaching. There is, thus a need to use visual content in order to enhance the learning experience of the students and supplement methods such as textbooks, on-line content, synchronous and asynchronous collaboration.

The user problem in modern education can be summarized as follows: learners need a service that improves efficiency in the cognitive and social domains: improve learning capacity and academic performance and increase group and individual self-confidence. There is a need to adopt experience - based e-learning services as an additional medium for engaging the students into actively taking part in distance learning. Furthermore, there is an important consideration that affects the design of any future service in Open Universities: the user population (students) can be measured in thousands. This means that any new service should be able to meet peak processing loads that may vary greatly over time. All of the above can be enabled by the utilization of Grid technologies.

\subsection{Possible learning examples in the near future}

Let us present two possible scenarios that are fully realizable with the utilization of Grid technologies in the near future. Although the scenarios are hypothetical, the technology and the infrastructure for their realization is available even today. Both are assuming the use of Grid technologies. 
In scenario 1, a learner (a high school student in this case) on an early Sunday morning is watching a documentary on television. The topic of the documentary is the solar planet system and the learner finds this quite interesting, so she desires to learn some more. A little later, while travelling on the back seat of her parents' car during a typical Sunday journey, she uses her palmtop to connect to her school's portal to use the Grid infrastructure and from there to NASA database to view some photos. These photos are actually located in a number of storage devises and in various physical locations, but the student views them as a unified collection available on the Grid. After viewing some photos of Mars (and perhaps after viewing Mars through a telescope connected to the Grid), she decides to create a simulation of the relative positions of Mars and Earth in our solar system. So she demands the appropriate service for this on the Grid and starts the simulation. While the student is watching the simulation on her palmtop, the computers from a number of school laboratories (closed on Sundays) -all connected to the Gridshare their CPU power to allow her to create all the data required for this service.

In scenario 2, a learner (a postgraduate student in Computer Science in this case) needs to complete an assignment on digital video watermarking. It is late on Sunday night and he needs to finish the assignment before Monday morning. So he uses his home computer and connects to the university portal to collect from the Grid a number of videos he could use for his assignment, without needing to worry about the actual location of all these videos. Then, he builds his own watermarking service by requiring from the Grid a number of available services (i.e. video coding, compression, etc.). In order to test his service, he starts applying his watermarking method on the videos. This time, utilizing the Grid infrastructure, the videos he processes are cut in small pieces and processed by a number of computers connected to the Grid that are in idle position during this time period. So the entire process takes a reasonable amount of time instead of hours. A similar scenario for using Grid technologies for digital images watermarking [4] has also been proposed.

These are just two possible scenarios that illustrate in a comprehensible way the utilization of Grid technologies in the everyday learning procedure. Of course, numerous other scenarios are realizable with the use of a learning Grid.

\section{THE HELLENIC OPEN UNIVERSITY EXPERIENCE}

\subsection{Presentation of HOU}

The Hellenic Open University supports a diverse population of students that register to undergraduate studies. Moreover, it provides postgraduate curricula to graduates who wish to extend or upgrade their studies to subjects related to their profession. Therefore, the HOU curricula correspond to various certificates, Bachelor or Master's degrees. A Bachelor degree may be comprised of several research directions.

Courses in the HOU are designed according to the distance learning methodology. According to this methodology, students study from distance using text books and digital educational material, participate in a number of tutorials (usually 5 per year for each module they attend) taking place in 8 towns, communicate with the corresponding tutor by telephone, fax, email and letters, prepare a number of written assignments (usually 4 to 6 for each module) and finally participate into a final examination at the end of each module. This examination takes place simultaneously in all major towns and the students have to be in specific locations and to be examined using the same examination sheets. Figure 2 shows the towns used as meeting points where the examination centers are located. Each student belongs to one student - group, called class. A class is located in one major Greek city in which class sessions take place. A tutor is allocated for each class of a maximum capacity of 32 students who inhabit in a specific geographical region. The academic personnel of the HOU includes a small number of permanent personnel as well as a large number of tutors. The permanent personnel undertakes, besides tutoring, the coordination of all classes and the overall academic responsibility for a specific course. Tutors cooperate with the $\mathrm{HOU}$ on an annual basis. They, in many cases, belong to the permanent staff of other Greek Universities.

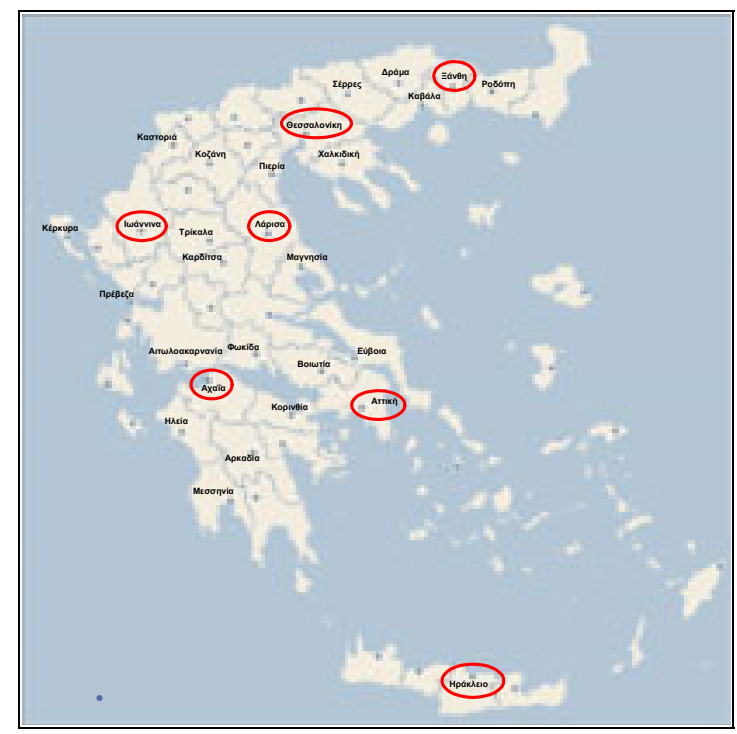

FIGURE 2. Major Greek towns used as meeting points 
The HOU allows admission of students without an entry examination. Although it is a public University, students pay fees for the cost of their studies. However, many students are supported by scholarships. The HOU, as an Open University, serves a diverse population of students. A typical HOU student is an adult (average age is just above 30), part time student. Many students are also professionals and have different cultural backgrounds and career goals. They are also geographically dispersed all around Greece.

\subsection{Distance education in the HOU}

During the academic year 2004-2005 the HOU provided a total of 6 courses leading to a Bachelor Diploma and 18 courses leading to a Masters Diploma. Approximately 20,000 students are currently attending the HOU courses. Most of the technologies and tools used for distance education presented in this paper were introduced in the Computer Science course of the School of Sciences and Technology of the HOU.

The Computer Science degree of $\mathrm{HOU}$ is a 4-year course that comprises 12 modules and leads to a Bachelor Diploma in Computer Science. Each student may register in one up to three modules per year. It should also be mentioned that each module is equivalent to 3 or 4 conventional university-level lessons. This Computer Science course has a high dropout rate [5], which is expected in distance education studies. The particularities and the nature of distance studies in the field of computer science, combined with the use of digital material in each course, provided perfect conditions for the introduction of distance education technologies and tools. In the following subsections, we present some of them. The steps taken by the HOU to implement and use these technologies are further analyzed in [6].

\subsubsection{Student's portal}

The HOU portal is used to distribute the digital material to students and to unify all services offered. The entry point is shown in figure 3. Each HOU student logs in and then has access only to the areas (modules) that (s)he attends.

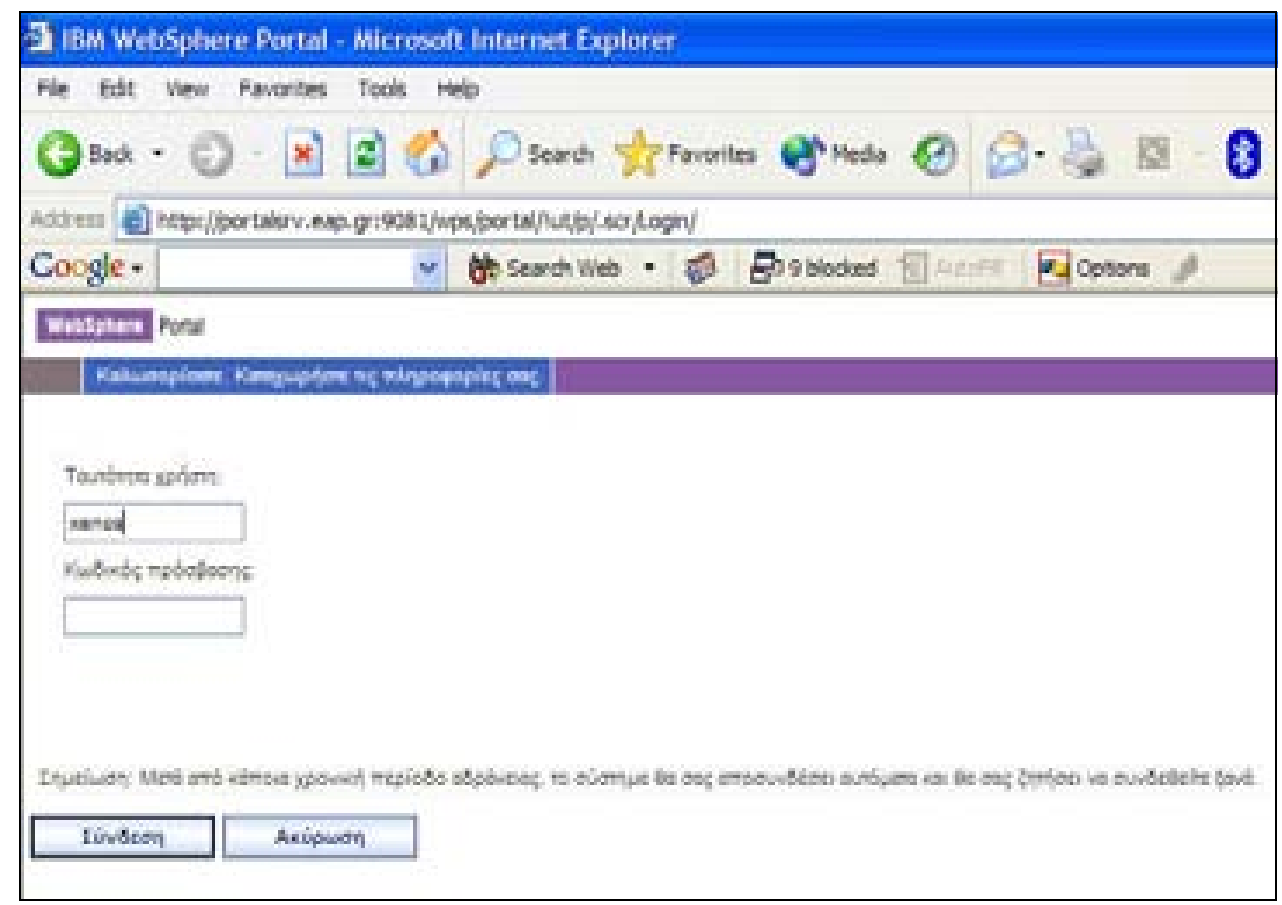

FIGURE 3. The entry point of HOU's portal

\subsubsection{Video Lectures}

A major part of the HOU's digital material that is available to students is the video lectures (also known as web casts). The HOU has started to produce several hundreds to several thousands of video-lectures. Such video lectures are small size (normally 5 to 15 minutes) videotaped lectures that combine a series of slides with narration and simple video (showing mostly the speaker).

The main goal of such video lectures is to clarify specific points of a course (usually spotted based on student queries). These lectures are made available through the web using streaming video technologies. Figure 4 illustrates an example of a Hellenic Open University video lecture explaining a particular case (i.e. the preorder traversal of binary trees) in the Computer Science course. Further details on the production of video lectures can be found in [7]. 


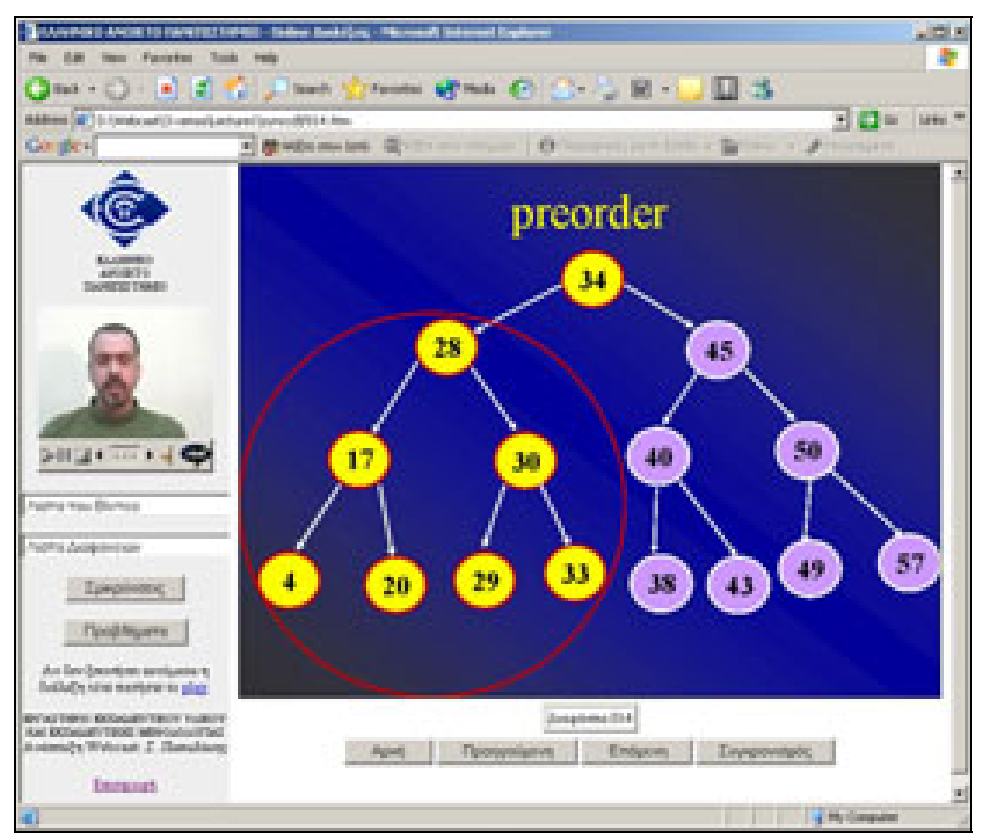

FIGURE 4. A video lecture used in the HOU

\subsubsection{Fora}

Although e-mail is commonly used mostly for non-public communication, i.e. for written assignment submission and corrections, the fora are the main tool for the communication among students and tutors. The tutors' forum proved to be valuable in coordinating this consulting effort, while students' fora were developed for specific groups (a forum for each class). Although the majority of students were in favor of using a forum, the students' fora were not at all successful. The success of a forum proved to be strongly related to group specificities and the tutorstudent preferred communication style. In many cases the use of e-mail and the provision of a group-specific web site including an announcement board (or a discussion list) was more effective than the forum.

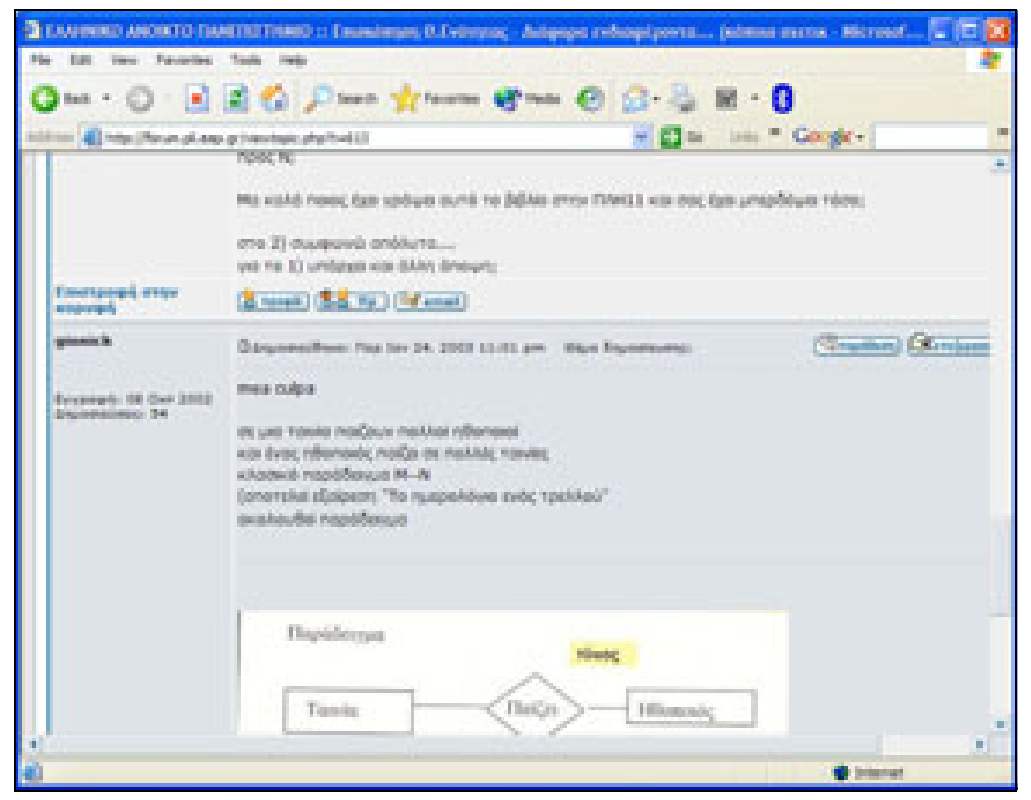

FIGURE 5. Discussion in a HOU's forum

In figure 5 an extract from a typical discussion in a forum of 25 first year students is shown. This is an example of an active forum with approximately 30 postings per week. In this figure a student (id: gionick) replies to the tutor's request by publishing an example (attached image).

\subsubsection{Virtual Classrooms}

Virtual classrooms are used in the HOU to emulate real classroom lectures. In such virtual classrooms students log on to the system and attend a lecture, while interacting with the tutor and with each other. Virtual classrooms allow students to write on the slides, to share their computer desktop or view, etc. Virtual classroom courses may be recorded and stored for playback. 
An example from a virtual classroom lecture in the Hellenic Open University is shown in figure 6 . This figure shows a virtual classroom with a tutor and 16 students. The tutor is explaining an algorithm, while a student (his microphone is highlighted) is asking a question using a red dot on the slides.

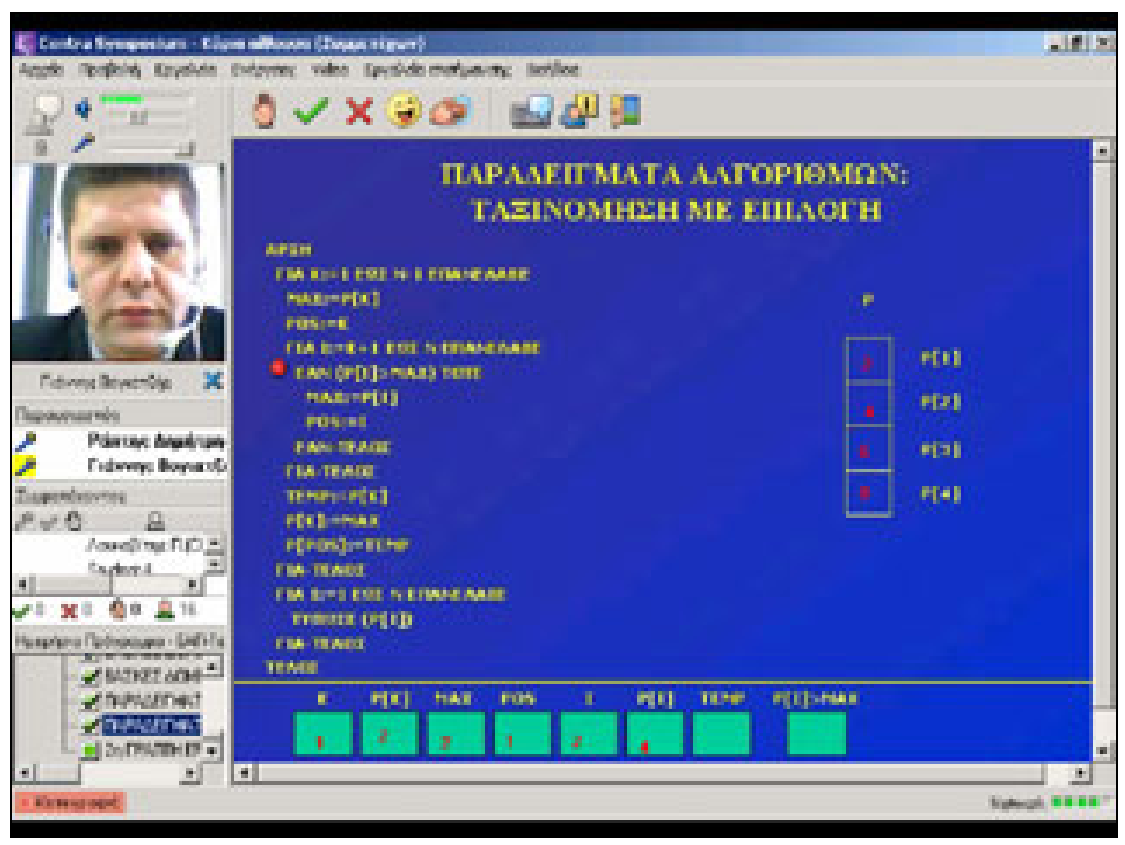

FIGURE 6. An extract from a virtual classroom

The use of virtual classrooms in the HOU lead to a major increase (up to $46 \%$ ) in the participation of remote students in meetings, but also caused some problems, as virtual classrooms could not fully replace actual classrooms and the students' 'feeling of participation' in them. On the other hand, it proved that it is definitely better to participate in a virtual classroom than to not participate at all, when actual meeting locations are out of reach. Another problem reported is that virtual meetings cannot follow a traditional meeting schedule. It is better to hold shorter but more frequent virtual classroom meetings than few long ones, as in the case of face-to-face meetings.

\subsubsection{E-Collaboration tools}

A number of synchronous collaborative problem solving activities have been introduced in the HOU. Specifically, groups of students work collaboratively from a distance in order to design the solution to a given problem. Figure 7 shows extracts from student solutions to collaborative building of an algorithm of a bus-ticket vending machine using a flowchart representation. Further details regarding the application of e-collaboration in the HOU can be found in [8].

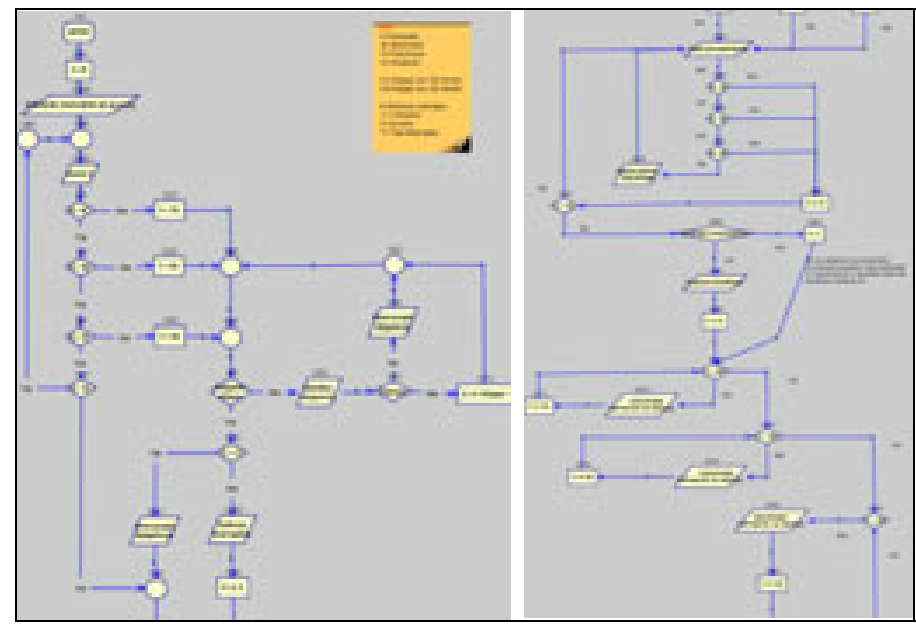

FIGURE 7. Extracts from student solutions

\subsection{The HOU Grid scenario}

The introduction of Grid technologies in the HOU's infrastructure must enable a leverage of the University's course administrative operations, providing new ways for communicating and introducing the use of improved teaching methodologies. However, this is not an easy task since it has to overcome the traditional ways of administration, information sharing, and teaching. Moreover, it needs an effective student-centered implementation and support mechanism in order to assure its widest acceptance and use by the academic community. It will integrate existing tools and databases that are already in use and possibly extend their capabilities. 
Another goal of the HOU is to improve collaboration between students by using Grid technologies. This will be accomplished through cooperative experiments. In a cooperative experiment, students share resources (data sets, results, opinions etc.) to perform a complex, multi step process.

Students may access the HOU service from anyplace in Greece through a portal. There are 8 points supporting the portal, called nodes, which are used for sharing resources and servicing users. Nodes are geographically dispersed and they are based in major Greek cities where HOU classes are making their regular sessions (see figure 8). Each node is covering a specific region and supports mainly the students that are living in the vicinity. The backbone that is used by the nodes for transferring data is the GRNet (Greek Universities Network), a highspeed network infrastructure that links all Greek higher education organizations. The speed of GRNet is currently at $155 \mathrm{Mbps}$. The goal of the HOU is to transform and extend the actual city-class model to a Grid model. Each node plays the role of an electronic regional support center, offering basic services such as direct and personalized attention towards students: virtual meetings with teachers and other students, seminars and round tables. There are two super nodes that are coordinating the smaller ones. Super nodes are used to handle large CPU loads, store intermediate results from simulation runs and backup data.

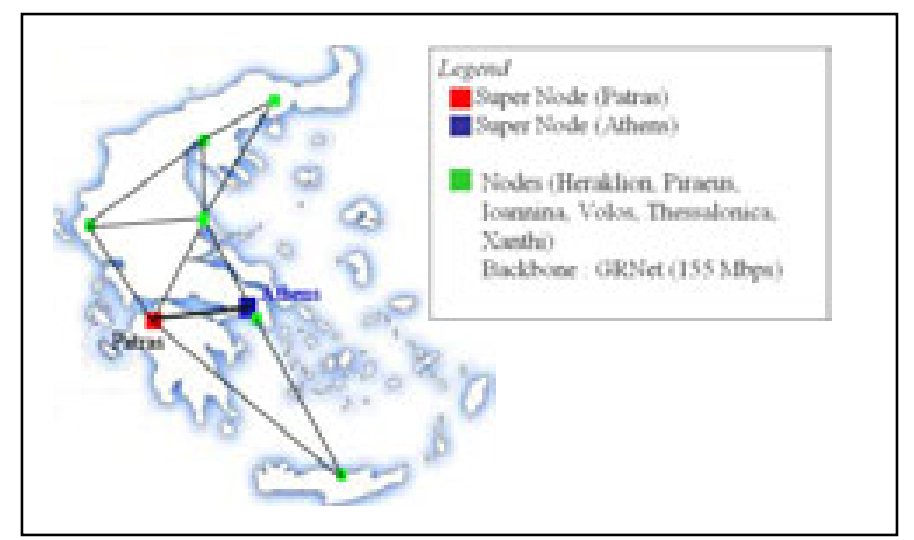

FIGURE 8. The nodes of the HOU Grid

Since the HOU's widespread deployment of computational resources involves the Internet as a communication backbone, the Grid infrastructure must seamlessly deal with the heterogeneous nature of the network. Some of the challenges that such an infrastructure must deal with are:

a) the user population is large and dynamic. Nevertheless, participants include members of the same institution,

b) the resource pool is large and dynamic, the quantity and location of available resources can change rapidly,

c) a computation (or process created by a computation) may acquire, start processes on, and release resources dynamically during its execution, and

d) resources have, in general, the same authentication and authorization mechanisms and policies.

Nevertheless, differences across different domains inside the HOU do exist (i.e. different laboratories).

As an outcome of the introduction of Grid technologies in the HOU, an advanced portal can be created that will cover a multitude of applications, and will constitute a single, consistent window through which the HOU information and applications can be accessed by the users (using the Grid infrastructure) in a personalized, webbased working environment. The HOU portal, therefore, will act as a unified access point through which users will share explicit information and exploit online collaboration tools with appropriate functionality (through the use of portal components) for the specific role of each user. This portal will support four different types of services (see figure 9):

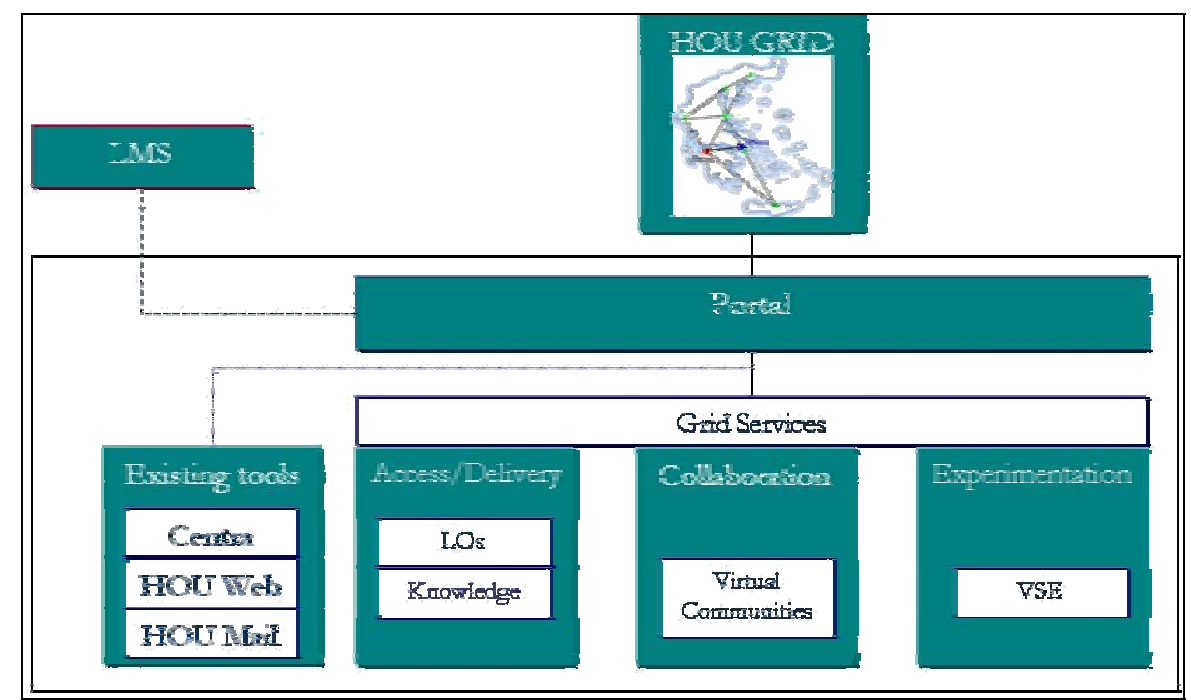

FIGURE 9. The HOU portal using Grid services 
1) collaboration and communication: using synchronous and asynchronous tools,

2) delivery: delivery of learning content,

3) operation: special tools used to generate material/communicate (e.g. MS Office tools),

4) experimentation: services for contacting experiments.

\section{CONCLUSION}

This paper discussed the potential created by Grid technologies in education and the way in which Grid technologies may affect the future of education. Still, unless education eventually moves towards other directions, it is almost certain that the current and future technological solutions will more and more turn traditional classroombased education into 'everywhere available' education. In such a learning paradigm, Grid technologies and learning Grids may play a very significant role. Of course, one needs to admit that, many times, technological breakthroughs surpass future estimations.

The case of the Hellenic Open University (HOU) presented in this paper is an actual case. The currently used educational technologies and tools were described and a scenario for the utilization of Grid technologies at HOU was presented. This scenario is already in the design phase and will be implemented in the near future.

\section{ACKNOWLEDGMENT}

The authors would like to acknowledge the support of the EC IP (Integrated Project, contract no IP002205) "ELeGI: European Learning GRID Infrastructure", 6th Framework Programme.

\section{REFERENCES}

[1] Foster I., Kesselman C., and Tuecke S., "The Anatomy of the Grid: Enabling Scalable Virtual Organizations", International Journal of High Performance Computing Applications, Vol. 15 (3), pp. 200-222, 2001.

[2] Open Grid Services Architecture (OGSA), http://www.ggf.org/ogsa-wg/

[3] Rold C., and Berg T., "Sourcing Strategies: Relationship Models and Case Studies", Note Number: R-18-9925, Gartner, Inc., Stanford, CT, 2003.

[4] Vassiliadis B., Fotopoulos V., Xenos M., Skodras A., "Could Grid Facilitate Demanding Media Watermarking Applications?", Proceedings of the 4th International LeGE-WG Workshop, Stuttgart, Germany, April, 2004.

[5] Xenos M., Pierrakeas C. and Pintelas P., "A Survey on Student Dropout Rates and Dropout Causes Concerning the Students in the Course of Informatics of the Hellenic Open University", Computers \& Education, Vol. 39 (4), pp. 361-377, 2002.

[6] Xenos M. and Skodras A., "Evolving from a Traditional Distance Learning Model to e-Learning", Proceedings of the 1st International LeGE-WG Workshop, Paris, France, 2003.

[7] Hadzilacos T., Kalles D., Papadakis S. and Xenos M., "Productionizing of Streaming Educational Material", Proceedings of the ICL International Workshop, Villach, Austria, 2003.

[8] Xenos M., Avouris N., Komis V., Stavrinoudis D. and Margaritis M., "Synchronous collaboration in distance education: a case study in a Computer Science Course", Proceedings of the 4th IEEE International Conference on Advance Learning Technologies, Joensuu, Finland, pp. 500-504, August 2004.

[9] Foster, C. Kesselman, J. Nick, and S. Tuecke. The Physiology of the Grid: An Open Grid Services Architecture for Distributed Systems Integration. Open Grid Service Infrastructure WG, Global Grid Forum, June 2002.

[10] D. Arnold, H. Casanova, and J. Dongarra. Innovation of the NetSolve Grid Computing System. Concurrency and Computation: Practice and Experience, 2003.

[11] G. Allen, T. Dramlitsch, I. Foster, N. Karonis, M. Ripeanu, E. Seidel, and B. Toonen. Supporting Efficient Execution in Heterogeneous Distributed Computing Evironments with Cactus and Globus. Proceedings of Supercomputing 2001, Nov. 2001 